Letters to the Editor

\title{
The Interdisciplinary Management of Newly Diagnosed Pituitary Tumors
}

by Dr. med. Cornelia Jaursch-Hancke, PD Dr. med. Timo Deutschbein, PD Dr. med. Ulrich J. Knappe,

Prof. Dr. med. Wolfgang Saeger, Prof. Dr. med. Jörg Flitsch, and Prof. Dr. med. Martin Fassnacht in issue 14/2021

\section{Further Interdisciplinary Considerations}

The authors succeeded in presenting a comprehensive overview of the diagnostic evaluation and surgical options (1). As an addendum, we wish to explain modern radiotherapeutic treatment options in combination with neurosurgical resection. Indications for radiotherapy include a (progressive) residual tumor residue, recurrence, or a high risk of re-operation and thus, radiotherapy is not a matter of routine postoperatively. In addition to conventional fractionated radiotherapy, the available radiation techniques are singledosestereotactic radiosurgery and fractionated stereotactic radiotherapy. Local control rates rates range between $72 \%$ and $100 \%$ after five years (2). Furthermore, after radiotherapy, normalization of the dysregulated hormonal axes is possible, as are improvements in visual acuity due to tumor regression $(2,3)$.

The radiotherapy concept and dose to be administered should be chosen depending on the size of the adenoma and the distance to critical organs at risk, especially the optic chiasm. Typical doses prescribed for conventional (normofractionated) radiotherapy are 45-54 Gy (Gray) in fractions of 1.8-2 Gy (3). By contrast, radiosurgery uses 13-16 Gy or 16-25 Gy for non-secreting or secreting adenomas, respectively (3). For the optimal administration of radiotherapy, precise planning imaging, exact contouring of the target volume and organs at risk, and the option of image guided radiotherapy are essential. The high local control rate has to be balanced against potential (long term) toxicities, such as hypopituitarism, which can affect $20-50 \%$

\section{In Reply:}

We welcome the comments from Oertel et al as a welcome addition to our article. They highlight additional therapeutic options in the treatment of previously operated pituitary adenomas, which we could not include in any detail in our article owing to space restrictions (1). The comments regarding radiotherapy for pituitary adenomas are consistent with the details and recommendations given in the clinical practice guideline entitled "Diagnostik und Therapie klinisch hormoninaktiver Hypophysentumoren [the diagnosis and treatment of clinically endocrine inactive pituitary tumors]" issued by the Association of the Scientific Medical Societies in Germany (Arbeitsgemeinschaft der Wissenschaftlichen Medizinischen Fachgesellschaften, AWMF) (2). Single stereotactic radiosurgery and of patients (2). Its occurrence is dose-dependent. In a modern radiosurgical collective, 11 Gy or more were identified as a dosimetric predictor (4). By contrast, the risk of radiogenic optic neuropathy is low, at $1-5 \%$ (2).

Overall, the risks and benefits of radiotherapy should be balanced for the individual patient. The neurosurgical and radiotherapeutic exchange required to achieve this, taking into account neuropathological and neuroradiological expertise, should ideally take place in the context of an interdisciplinary tumor board.

DOI: 10.3238/arztebl.m2021.0255

\section{References}

1. Jaursch-Hancke C, Deutschbein T, Knappe UJ, Saeger W, Flitsch J, Fassnacht M: The interdisciplinary management of newly diagnosed pituitary tumors. Dtsch Arztebl Int 2021; 118: 237-43.

2. Chanson $P$, Dormoy A, Dekkers OM: Use of radiotherapy after pituitary surgery for non-functioning pituitary adenomas. Eur J Endocrinol 2019; 181: D1-13

3. ESTRO ACROP guideline for target volume delineation of skull basetumors. Radiother and Oncol 2021; 56: 80-94

4. Graffeo CS, Link MJ, Brown PD, Young WF, Pollock BE: Hypopituitarism after single-fraction pituitary adenoma radiosurgery: dosimetric analysis based on patients treated using contemporary techniques. Int J Radiat Oncol Biol Phys 2018; 101: 618-23.

\section{Dr. med. Michael Oertel}

Klinik für Strahlentherapie - Radioonkologie

Universitätsklinikum Münster (UKM)

michael.oertel@ukmuenster.de

PD Dr. med Eric Suero Molina

Prof. Dr. med. Walter Stummer

Klinik für Neurochirurgie

Universitätsklinikum Münster (UKM)

Prof. Dr. med. Hans Theodor Eich

Klinik für Strahlentherapie - Radioonkologie

Universitätsklinikum Münster (UKM) (hypo)fractionated radiotherapy have their place in treating pituitary adenomas, depending on the respective size and contour of the postoperative residual tumor and its relation to the anterior visual pathway. The advantages of radiosurgery over fractionated radiotherapy in endocrine active tumors-for example, in acromegaly - are the tendentially faster effect on the hypersecretion syndrome and the significantly lower rate of secondary pituitary dysfunction (3), when-as Oertel and colleagues correctly pointed out - much higher dosages have to be administered than for endocrine inactive tumors. For this reason, the initially interdisciplinary approach makes sense-namely, to undertake individually tailored partial tumor resection in order to enable radiosurgical treatment - in treating pituitary adenomas that are not completely resectable. 
Under the label "separation surgery" this has also been proposed for treating vertebral body metastases (4). We agree with our correspondents that therapeutic decisions in this tumor entity too should be made in the setting of interdisciplinary tumor boards (2).

DOI: 10.3238 /arztebl.m2021.0256

\section{References}

1. Jaursch-Hancke C, Deutschbein T, Knappe UJ, Saeger W, Flitsch J, Fassnacht $\mathrm{M}$ : The interdisciplinary management of newly diagnosed pituitary tumors. Dtsch Arztebl Int 2021; 118: 237-43.

2. Deutschbein T, Jaursch-Hancke C, Knappe UJ, et al.: First German guideline on diagnostics and therapy of clinically non-functioning pituitary tumors. Exp Clin Endocrinol Diabetes 2021; 129: 250-64.
3. Knappe UJ, Petroff $D$, Quinkler $M$, et al.: Fractionated radiotherapy and radiosurgery in acromegaly: analysis of 352 patients from the German acromegaly registry. Eur J Endocrinol 2020; 182: 275-84.

4. Bate BG, Khan NR, Kimball BY, et al.: Stereotactic radiosurgery for spinal metastases with or without separation surgery. J Neurosurg Spine 2015; 22: 409-15

On behalf of the authors

PD Dr. med. Ulrich Knappe

Prof. Dr. med. Jörg Flitsch

Endokrinologie, DKD HELIOS Klinik Wiesbaden

jaurschhancke@icloud.com

\section{Conflict of interest statement}

The authors of both contributions declare that no conflict of interest exists.

\section{-O) CLINICAL SNAPSHOT}

\section{Bilateral Plantar Phlebothrombosis After AstraZeneca Vaccination}
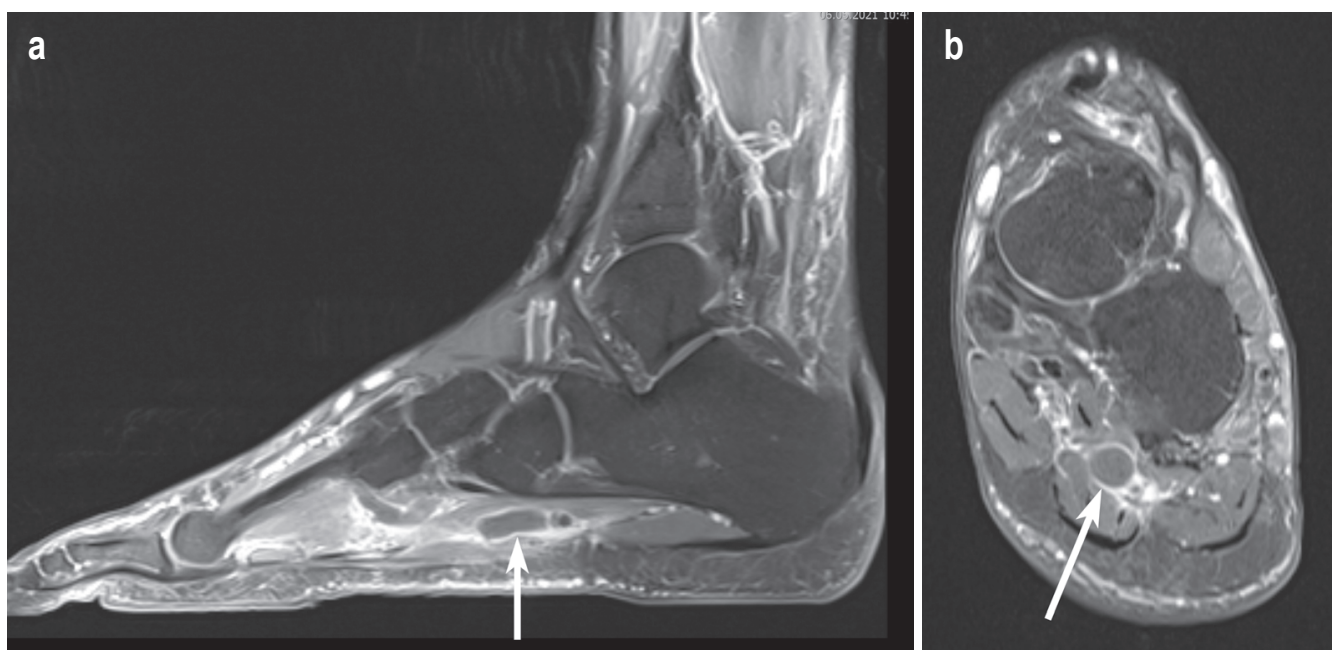

MRI:

a) Sagittal and

b) frontal T1-weighted fat-saturated sequence following intake of contrast medium, showing characteristic non-enhancement of the plantar venous arch

A 63-year-old, otherwise healthy man who was working at home due to the COVID-19 pandemic suffered intense pain and cramp in the sole of his left foot. His primary care physician referred him for exclusion of a symptomatic heel spur. However, magnetic resonance imaging (MRI) detected a non-enhancing phlebothrombosis of the plantar venous arch over $10 \mathrm{~cm}$ long (Figure). The patient had no history of thromboses, but reported that the pain in his left foot had begun 6 days after his first dose of the AstraZeneca vaccine, while the less pronounced pain in the right foot had begun 3 days after the injection. MRI of the right foot was then performed and a 4-cm-long partial thrombosis was found. The angiologist who we then consulted first confirmed the diagnosis by means of D-dimer determination (1.3) and Doppler ultrasonography and then administered the fast-acting anticoagulant apixaban (Eliquis). The D-dimer level 2 weeks later was 0.6 , and the patient is currently free of symptoms. Although association with the vaccine cannot be proven, this case shows that besides the rarely occurring venous sinus thrombosis, thromboses at other, perhaps unexpected sites must also be considered as potential causes of pain following vaccination against SARS-CoV-2.

Dr. Sebastian Lins, MRT Radiologie am Gasteig, München, info@radiologe-in-muenchen.de

Alice Kolbinger, Allgemeinmedizin Josephsburg, München

Dr. Karl Ludwig Maier, Internist \& Angiologe Moosfeld, München

Conflict of interest statement: The authors declare that no conflict of interest exists.

Translated from the original German by David Roseveare

Cite this as: Lins S, Kolbinger A, Maier KL: Bilateral plantar phlebothrombosis after AstraZeneca vaccination.

Dtsch Arztebl Int 2021; 118: 511. DOI: 10.3238/arztebl.m2021.0273. 